\title{
Compact optical temporal processors
}

\author{
David Mendlovic, Oded Melamed, and Haldun M. Ozaktas
}

\begin{abstract}
Optical signal processing can be done with time-lens devices. A temporal processor based on chirp-z transformers is suggested. This configuration is more compact than a conventional $4-f$ temporal processor. On the basis of implementation aspects of such a temporal processor, we did a performance analysis. This analysis leads to the conclusion that an ultrafast optical temporal processor can be implemented.
\end{abstract}

\section{Introduction}

A signal processor is the main component of many digital or analog computers. The computing rate of the processor may determine the speed of the whole system. Recently, several optical systems were suggested $^{1-3}$ that can achieve ultrafast serial processing by employment of the analogy between optical spatial diffraction and temporal dispersion. ${ }^{4-6}$ In the following, a temporal processor that is based on a chirp-z transformer is suggested. As the first step toward practical implementation of the suggested system, its performance analysis was done. This analysis takes into account the state-of-the-art option for the timelens device. In Section 2, for the sake of notation, the relevant background is briefly presented.

\section{Optical Temporal Processor Based on Time Lenses}

In this section we summarize basic concepts in the theory of optical temporal processing based on timelenses. A detailed description of this theory is given in Ref. 2.

A monochromatic field distribution $u(x, z=0)$ with spatial spectrum $U(\mu)$ that travels along the $z$ axis will experience spatial diffraction according to Fresnel theorem:

$$
\begin{aligned}
u(x, z \geq 0) \simeq & C \exp (i k z) \int U(\mu) \exp \left(-i \pi \lambda z \mu^{2}\right) \\
& \times \exp (i 2 \pi \mu x) \mathrm{d} \mu
\end{aligned}
$$

D. Mendlovic and O. Melamed are with the Faculty of Engineering, Tel Aviv University, Tel Aviv 69978, Israel. H. M. Ozaktas is with Department of Electrical Engineering, Bilkent University, Bilkent 06533, Ankara, Turkey.

Received 7 July 1994; revised manuscript received 18 January 1995.

0003-6935/95/204113-06\$06.00/0.

(1) 1995 Optical Society of America.
The term $\exp \left(-i \pi \lambda z \mu^{2}\right)$ is known as the spatial dispersion term. $\quad C$ is a complex constant.

A temporal signal $u(t, z=0)$ with a spectrum $U(\omega)$ that travels along the $z$ axis of a dispersive delay line will accumulate phase delay

$$
\exp \{i 2 \pi[\omega t-\beta(\omega) z]\}
$$

where $\omega$ is the temporal analog of $\mu$ and $\beta(\omega)$ is commonly approximated as

$$
\beta(\omega) \approx \beta_{0}+\beta_{1} \omega+\beta_{2}\left(\omega^{2} / 2\right) .
$$

$\beta_{1}$ is the group velocity of the propagated wave and $\beta_{2}$ is the quadratic dispersion term. If we neglect irrelevant factors, the propagated signal from $z=0$ to $z$ is

$$
u(t, z)=\int_{-\infty}^{\infty} U(\omega) \exp \left(-i \pi \beta_{2} z \omega^{2}\right) \exp \left[i 2 \pi \omega\left(t-\beta_{1} z\right)\right] \mathrm{d} \omega
$$

Comparison of approximation (1) and Eq. (4) leads to the similarity between the spatial dispersion term $\exp \left(-i \pi \lambda z \mu^{2}\right)$ and the temporal dispersion factor $\exp \left(-i \pi \beta_{2} z \omega^{2}\right)$. This similarity is the basic motivation for developing such optical temporal processors. For the general convolution processor the temporal analogs for the spatial 2-f and 4- $f$ systems were designed. ${ }^{2}$ The basic components of these spatial systems (Fig. 1) are (1) free-space propagation, (2) spatial lenses, and (3) spatial filters. According to the above duality, one can define the appropriate components of the temporal $2-f$ and $4-f$ processors: (1) dispersive delay lines, (2) time-lenses, ${ }^{6,7}$ and (3) temporal filters.

Figure 2 shows a schematic $2-f$ system that performs the temporal Fourier-transform operation. It consists of propagation in a dispersive line along a length $f$, passage through a time lens, and again 


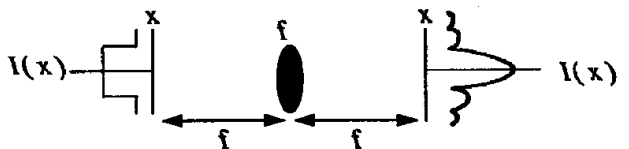

(a)

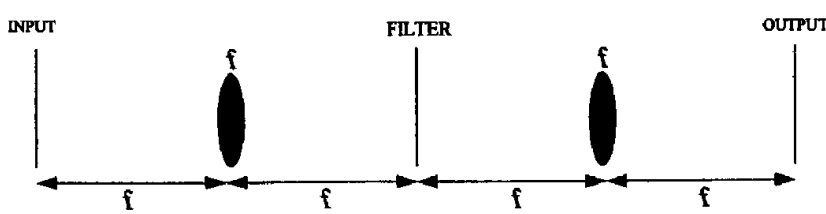

(b)

Fig. 1. Spatial processors: (a) 2-f system; the output of this system is a scaled Fourier transform of the input image. (b) 4- $f$ system; this is a basic Fourier optics image processor, which contains two 2- $f$ systems in cascade. (b) is a 1:1 imaging setup capable of Fourier-plane filtering.

propagation in another dispersive line along a length $f$. The time lens is described by the phase factor

$$
L(t)=\exp \left[-i \pi\left(\frac{t-t_{1}}{\tau}\right)^{2}\right],
$$

where $\tau$ and $t_{1}$ are the time-lens parameters that control the lens performance. ${ }^{2} \tau$ relates to the focal length, and $t_{1}$ relates to the lateral location of the lens in the spatial analog system. It was shown ${ }^{2}$ that for achieving the $2-f$ system, $\tau$ and $t_{1}$ should satisfy the following equations:

$$
\begin{aligned}
\tau^{2} & =\beta_{2} f, \\
t_{1} & =\beta_{1} f
\end{aligned}
$$

A time lens can be implemented either as an electrooptic phase modulator 7,8 or as an electro-optic amplitude modulator driven by an electrical chirp signal.

If the input signal at $z=0$ is $u_{0}(t)=u(t, 0)$, the output signal of the $2-f$ temporal processor ${ }^{2}$ is

$$
u(t, 2 f)=U_{0}\left(\frac{t-2 f \beta_{1}}{\tau^{2}}\right),
$$

where $U_{0}(\omega)$ is the Fourier transform of $u_{0}(t)$. Now let us expand the 2-f Fourier transformer to the basic Fourier-optics processor. The $4-f$ configuration, which contains two 2- $f$ systems in cascade (Fig. 3 ), is a $1: 1$ imaging setup capable of Fourier-plane filtering. The 4-f configuration includes two lenses. The first lens parameters are those that we calculated earlier for the 2-f system. The second lens ${ }^{2}$ has the same focal length as the first lens,

$$
\tau^{2}=\beta_{2} f
$$

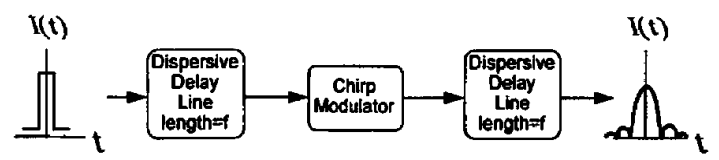

Fig. 2. Optical temporal 2- $f$ system.

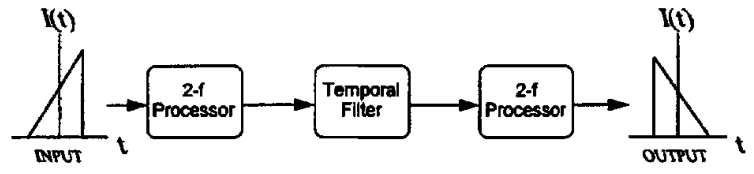

Fig. 3. Optical temporal 4-f processor.

but $t_{1}$ is changed to

$$
t_{1}=3 \beta_{1} f \text {. }
$$

For these parameters the output signal is

$$
u(t, 4 f)=u_{0}\left(4 f \beta_{1}-t\right) .
$$

It is exactly a delayed reverse 1:1 image of the input signal; i.e., the front and the end of the image are exchanged.

Implementation of such a processor requires four dispersive delay lines with length $f$ and four electrooptic modulators: one to modulate the input signal, one for each time lens, and one for Fourier-plane filtering. In Section 3 an alternative approach is suggested that requires only three electro-optic modulators and two dispersive delay lines.

\section{Temporal Processors Based on the Chirp-z Transformation}

\section{A. Temporal Chirp-z Transformer}

As in spatial optics, the use of chirp-z transformation could provide several benefits. Figure $4(\mathrm{a})$ shows a schematic spatial processor that performs a spatial Fourier transform. The setup is based on an optical implementation of the chirp-z transformation. ${ }^{9}$ It consists of three subsystems in cascade: transfer through a lens, free-space propagation, and again transfer through a lens. For the temporal dual case we multiply the input temporal signal with a time lens, then we let it propagate over a distance $f$, and finally we multiply it with another time lens [Fig. 4(b)].

Now let us start with an initial signal $u(t)$ with temporal spectrum $U(\omega)$. Just after the first time lens $L_{1}(t)$, the signal is

$$
u\left(t, 0^{+}\right)=u(t) L_{1}(t)
$$

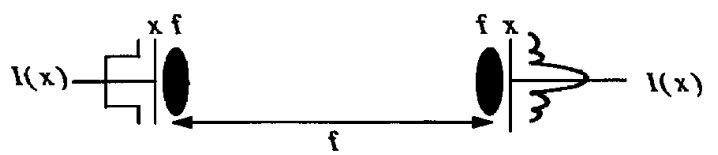

(a)

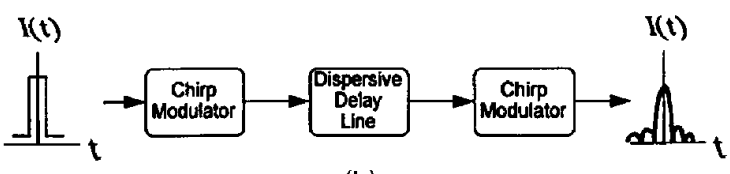

(b)

Fig. 4. Chirp-z transformer: (a) spatial configuration, (b) temporal analog configuration. 
$\mathrm{L}_{1}(t)$ is selected as

$$
L_{1}(t)=\exp \left(-i \pi \frac{t^{2}}{\beta_{2} f}\right)
$$

and its Fourier transform is

$$
L_{1}^{f}(\omega)=\left(\beta_{2} f\right)^{1 / 2} \exp (-i \pi / 4) \exp \left(i \pi \beta_{2} f \omega^{2}\right) .
$$

For the sake of simplification we prefer to express $u\left(t, 0^{+}\right)$by the Fourier integrals of $u(t)$ and $L_{1}(t)$ :

$$
u\left(t, 0^{+}\right)=\iint_{-\infty}^{\infty} U(\omega) L_{1}^{f}(\mu) \exp (i 2 \pi \omega t) \exp (i 2 \pi \mu t) \mathrm{d} \omega \mathrm{d} \mu .
$$

Just before the second time lens $L_{2}(t)$, the signal is

$$
\begin{aligned}
u\left(t, f^{-}\right)= & \iint_{-\infty}^{\infty} U(\omega) L_{1}^{f}(\boldsymbol{\mu}) \exp \{i \phi[(\mu+\omega), f]\} \\
& \times \exp (i 2 \pi \omega t) \exp (i 2 \pi \mu t) \mathrm{d} \omega \mathrm{d} \mu \\
\phi(\omega, f) \equiv & -2 \pi \beta_{1} f \omega-\pi \omega^{2} \beta_{2} f .
\end{aligned}
$$

By using one of the definitions of the delta function, we can rewrite Eq. (16) as

$$
u\left(t, f^{-}\right)=C U\left(\frac{t-\beta_{1} f}{\beta_{2} f}\right) \exp \left[i \pi \frac{\left(t-\beta_{1} f\right)^{2}}{\beta_{2} f}\right],
$$

where $C$ is an irrelevant constant. If $L_{2}(t)$ is chosen to be

$$
L_{2}(t)=\exp \left[-i \pi \frac{\left(t-\beta_{1} f\right)^{2}}{\beta_{2} f}\right],
$$

then just after the second lens, the signal is

$$
u(t, f)=C U\left(\frac{t-\beta_{1} f}{\beta_{2} f}\right),
$$

One can see from Eq. (19) that the output of the suggested system is a scaled and delayed version of the temporal Fourier transform of the input signal.

\section{B. Temporal Filtering System Based on Chirp-z Transformers}

The spatial imaging processor that is capable of Fourier-plane filtering is shown in Fig. 5(a). The temporal analog case is depicted in Fig. 5(b). It consists of two chirp-z transformers placed in cascade. In the following this configuration is denoted as a 2-chirp-z processor. The input signal for the second transformer is expressed in Eq. (19). Just after $L_{3}$, the signal is

$$
u\left(t, f^{+}\right)=U\left(\frac{t-\beta_{1} f}{\beta_{2} f}\right) L_{3}(t) .
$$

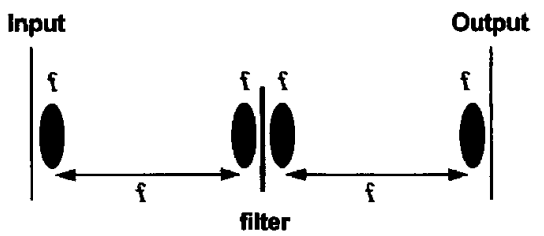

(a)

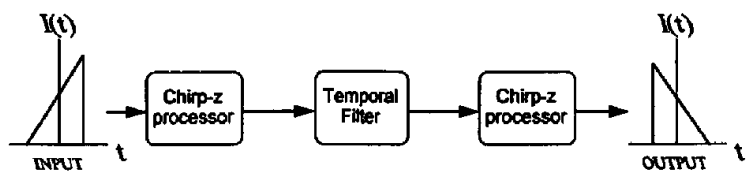

(b)

Fig. 5. 2-Chirp-z processor: (a) spatial configuration, (b) temporal analog configuration.

If $L_{3}(t)$ is

$$
L_{3}(t)=\exp \left[-i \pi \frac{\left(t-\beta_{1} f\right)^{2}}{\beta_{2} f}\right],
$$

$u\left(t, f^{+}\right)$can be rewritten as

$$
\begin{gathered}
u\left(\bar{t}, f^{+}\right)=U\left(\frac{\bar{t}}{\beta_{2} f}\right) \exp \left[-i \pi \frac{\bar{t}^{2}}{\beta_{2} f}\right] \\
\bar{t} \stackrel{\operatorname{def}}{=} t-\beta_{1} f .
\end{gathered}
$$

Because Eq. (22) is very similar to Eq. (12) except that $u(t)$ is replaced by $U\left(\bar{t} / \beta_{2} f\right)$, one can find from Eq. (17) that the signal just before the last lens $L_{4}(t)$ is

$$
u\left(t, 2 f^{-}\right)=u\left(2 \beta_{1} f-t\right) \exp \left[i \pi \frac{\left(t-2 \beta_{1} f\right)^{2}}{\beta_{2} f}\right] .
$$

If $L_{4}(t)$ is selected as

$$
L_{4}(t)=\exp \left[-i \pi \frac{\left(t-2 \beta_{1} f\right)^{2}}{\beta_{2} f}\right],
$$

the output signal is

$$
u(t, 2 f)=u\left(2 \beta_{1} f-t\right) .
$$

The advantage of using such a processor instead of a conventional 4- $f$ processor is that it can be implemented with only two dispersive delay lines and three electro-optic modulators: the first modulator modulates the input signal multiplied by the first time lens, the second implements the second and the third time lenses $\left[L_{2}(t)\right.$ and $\left.L_{3}(t)\right]$, and the third implements the last time lens $\left[L_{4}(t)\right]$. In many applications, $u(t)$ is real and positive. In such cases the third modulator is not required, and the system can be implemented with only two electro-optic modulators.

\section{Performance Analysis}

\section{A. The Chirp-z Fourier Transformer}

For estimating the performance of the chirp-z temporal configuration two considerations are used. The first is to prevent any noncausal information along 


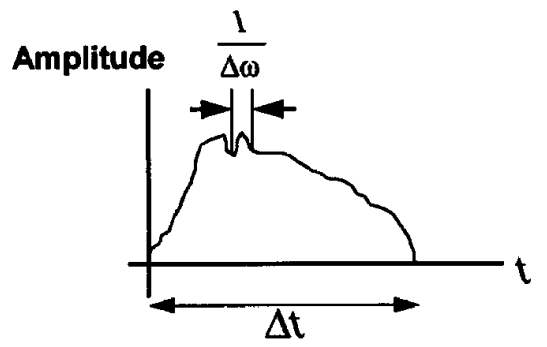

Fig. 6. Schematic illustration of the input temporal signal with a total width of $\Delta t$ and a finest resolution of $1 / \Delta \omega$.

the signal flow. Second, overlapping between the signal and its processed version is not possible.

Figure 6 shows the input signal with a width of $\Delta t$ and finest resolution of $1 / \Delta \omega(\Delta \omega$ is the signal bandwidth). To find the signal's width $\Delta t_{1}$ after propagation in a dispersive line with a length $f$, we first discuss the effect of group-velocity dispersion on a Gaussian pulse ${ }^{10}$ :

$$
s(t, z=0)=\exp \left(-t^{2} / 2 T_{0}^{2}\right) .
$$

$T_{0}$ is the half-width (at the $1 / e$ intensity point). By using Eq. (4) and carrying out the integration, we can obtain the amplitude at any point $z$ :

$$
s(t, z)=\left(\frac{T_{0}^{2}}{T_{0}^{2}-i \beta_{2} z}\right)^{1 / 2} \exp \left[-\frac{\left(t-\beta_{1} z\right)^{2}}{2\left(T_{0}^{2}-i \beta_{2} z\right)}\right] .
$$

The width of the Gaussian pulse increases and becomes

$$
T_{1}=T_{0}\left[1+\left(\frac{z \beta_{2}}{T_{0}^{2}}\right)^{2}\right]^{1 / 2} .
$$

If $\left(z \beta_{2} / T_{0}^{2}\right)^{2} \gg 1$, Eq. $(28)$ can be rewritten as

$$
T_{1}=z \beta_{2} / T_{0} .
$$

The input signal $u(t)$ can be considered as composed of NPs (NPs $\gg 1)$ resolution points, each with a Gaussian shape and half-width $T_{0}=2 / \Delta \omega$ (Fig. 7). The signal's widening is thus $2 T_{1}$, and its width just before the second lens at $z=f^{-}$becomes

$$
\Delta t_{1}=\Delta t+\beta_{2} f \Delta \omega .
$$

In analogy with a spatial processor, $\Delta t_{1}$ is the required second-lens aperture, an important parameter that affects the system's time-bandwidth-product performance. To prevent noncausal signals, we have to

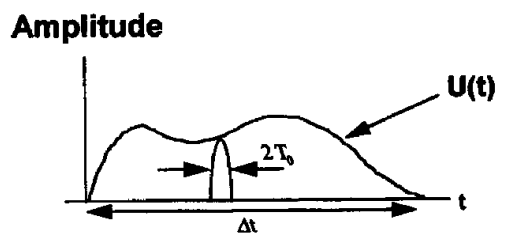

Fig. 7. Input signal is composed of NPs resolution points, each with a Gaussian shape and half-width $T_{0}$. assume that

$$
\Delta t_{1}<\beta_{1} f,
$$

where $\beta_{1} f$ is the propagation time from the input plane to the second lens. To prevent overlapping (Fig. 8), we should make the time distance between two successive signals

$$
\delta T>\Delta t_{1} .
$$

As Eq. (19) shows, the output signal of the chirp-z transformers is a scaled Fourier transform of the input signal. The temporal width of the output signal $\Delta t_{2}$ is thus

$$
\Delta t_{2}=\beta_{2} f \Delta \omega .
$$

The spectrum of the output signal can be determined from

$$
\mathrm{FT}[u(t, f)]=\mathrm{FT}\left[U\left(\frac{t-f \beta_{1}}{\tau^{2}}\right)\right]=C u\left(-\beta_{2} f \omega\right) .
$$

FT indicates the Fourier transform, and $C$ is an irrelevant constant. The spectral width of the output signal is

$$
\Delta \omega_{2}=\Delta t / \beta_{2} f
$$

For achieving temporal filtering a temporal filter should be used in the Fourier plane. Its temporal width, $\Delta t_{F}$, and spectral width, $\Delta \omega_{F}$, are

$$
\begin{gathered}
\Delta t_{F}=\Delta t_{2}, \\
\Delta \omega_{F}>\Delta t / \beta_{2} f .
\end{gathered}
$$

Expressions $(30)-(37)$ state all restrictions on the chirp-z Fourier transformer and permit the calculation of the system's performance.

Expressions (30) and (31) can be reduced to

$$
\Delta t<f\left(\beta_{1}-\beta_{2} \Delta \omega\right) .
$$

Because $\Delta t>0$, inequality (38) leads to the conclusion that, when a dispersive line is chosen, the following relation between the group velocity and the dispersion term must exist

$$
\Delta \omega<\beta_{1} / \beta_{2} .
$$

If $\Delta \omega_{M}$ is the maximum bandwidth of the available

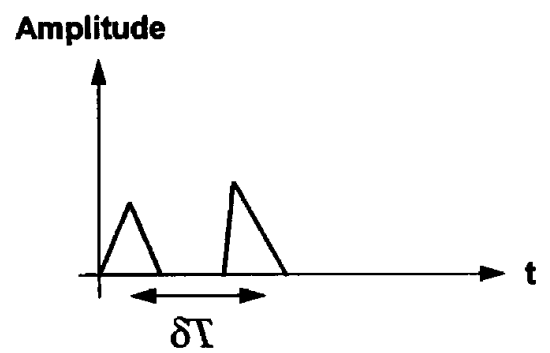

Fig. 8. Time distance between two input signals. 
modulator, and assuming that $\Delta \omega=\Delta \omega_{F}=\Delta \omega_{M}$, Eq. (37) can be rewritten as

$$
\Delta t<\Delta \omega_{M} \beta_{2} f .
$$

The system is a serial processor in first-in first-out configuration. The processor's input are signals with a frame width of $\Delta t$ that enter the system, one after another, every $\delta T$. The suggested configuration, consisting of a modulator with a bandwidth of $\Delta \omega_{M}$, can process $\mathrm{NP}_{\mathrm{s}}$ points (or pixels) every signal frame, where $\mathrm{NP}_{\mathrm{s}}$ is given by

$$
\mathrm{NP}_{\mathrm{s}}=\Delta t \Delta \omega_{M},
$$

The restrictions on the signal's width $\Delta t$ are given in inequalities (38) and (40) and can be rewritten, respectively, as

$$
\begin{aligned}
& \Delta t=\left(1 / c_{1}\right) f\left(\beta_{1}-\beta_{2} \Delta \omega\right), \quad c_{1}>1, \\
& \Delta t=\left(1 / d_{1}\right) \Delta \omega_{M} \beta_{2} f, \quad d_{1}>1,
\end{aligned}
$$

where $c_{1}$ and $d_{1}$ are constants determined by the system's designer. The signal's width is determined by the more limiting expression, i.e., the one that causes the frame width $\Delta t$ to be smaller. Substitution of Eqs. (42) and (43) into Eq. (41) yields

$$
\begin{array}{r}
\mathrm{NP}_{\mathrm{s}}=\operatorname{MIN}\left[\left(1 / c_{1}\right) f\left(\beta_{1}-\beta_{2} \Delta \omega_{M}\right) \Delta \omega_{M}, \Delta \omega_{M}^{2} \beta_{2} f\left(1 / d_{1}\right)\right], \\
c_{1}, d_{1}>1 .
\end{array}
$$

MIN is for the minimum function that chooses the most limiting expression in the square brackets.

Because the system processes $1 / \delta T$ frames every second, the total number of processed points per second is determined by

$$
N=\Delta t \Delta \omega_{M} / \delta T .
$$

By using expressions (30) and (32), we can write $\delta T$ as a function of the signal's width $\Delta t$ :

$$
\delta T>\Delta t+\beta_{2} f \Delta \omega_{M} .
$$

If the restrictions imposed on $\Delta t[$ Eqs. (42) and (43)] are substituted into inequality (46), then this inequality can be rewritten as

$$
\delta T=c_{2}\left[\left(1 / c_{1}\right) f\left(\beta_{1}-\beta_{2} \Delta \omega_{M}\right)+f \beta_{2} \Delta \omega_{M}\right], \quad c_{2}>1,
$$

or

$$
\delta T=d_{2}\left[\left(1 / d_{1}\right) f \beta_{2} \Delta \omega_{M}+f \beta_{2} \Delta \omega_{M}\right], \quad d_{2}>1 .
$$

Equations (47) and (48) are related to the restrictions expressed in Eqs. (42) and (43), respectively. $\quad c_{2}$ and $d_{2}$ are constants that are chosen by the system's designer and actually replace the inequality sign in inequality (46). Therefore the total number of points processed by the system every second, $N$, can be calculated by

$$
\begin{aligned}
N=\operatorname{MIN}\left\{\frac{\left(\beta_{1}-\beta_{2} \Delta \omega_{M}\right) \Delta \omega_{M}}{c_{2}\left[\beta_{1}+\beta_{2} \Delta \omega_{M}\left(c_{1}-1\right)\right]}, \frac{\Delta \omega_{M}}{d_{2}\left(d_{1}+1\right)}\right\}, \\
c_{1}, c_{2}, d_{1}, d_{2}>1 .
\end{aligned}
$$

In conclusion of this section, Eq. (49) shows that proper design of the system can accelerate the process up to a rate of $\Delta \omega_{M} / 2$ points per second.

\section{B. 2-Chirp-z Processor}

The 2-chirp-z processor can be separated into two chirp-z subsystems in cascade. The first chirp-z Fourier transformer was analyzed in Subsection 4.A. The second Fourier transformer is analyzed similarly but with different input parameters. The input signal is

$$
u(t, 2 f)=U\left(\frac{t-2 f \beta_{1}}{\beta_{2} f}\right),
$$

and its temporal and spectral widths, respectively, are

$$
\begin{aligned}
\Delta t_{2} & =\beta_{2} f \Delta \omega, \\
\Delta \omega_{2} & =\Delta t / \beta_{2} f .
\end{aligned}
$$

The analysis of the whole system leads to the conclusion that no further restrictions are added by the second transformer, and thus the number of processed points per second is identical whether the system consists of one or two chirp-z transformers.

\section{Typical Example}

In this subsection we discuss a typical example of a temporal processor with a time lens. The input signal and the time lens are created with an electrooptic modulator. Present technology offers modulators with $\Delta \omega_{M}=30 \mathrm{GHz}$. A dispersive delay line is used with $\beta_{2}=4.5 \times 10^{-20} \mathrm{~s}^{2} / \mathrm{m}, \beta_{1}=5 \times 10^{-9} \mathrm{~s} / \mathrm{m}$, and $f=50 \mathrm{~m}$. Such dispersion can be achieved in optical fiber filters. ${ }^{11}$ The input width is chosen to be $\Delta t=3.4 \times 10^{-8} \mathrm{~s}$, and the time distance between two successive signals is $\delta T=2 \times 10^{-7} \mathrm{~s}$. According to Eq. (41), this system can process approximately 1020 points per session and a total of $5 \times 10^{6}$ sessions per second.

\section{Conclusions}

An optical temporal filtering processor based on chirp-z transformers has been analyzed. Because the implementation of optical processors based on a time lens is very complicated and expensive, there is a great importance in reducing the number of components of the system. The suggested system is more compact than a conventional 4- $f$ setup because it requires only two dispersive delay lines with a length $f$ and three electro-optics modulators at most. A performance analysis was done to motivate further research in implementing the suggested system. 


\section{References}

1. A. W. Lohmann and D. Mendlovic, "Temporal perfect-shuffle optical processor," Opt. Lett. 17, 882-884 (1992).

2. A. W. Lohmann and D. Mendlovic, "Temporal filtering with time lenses," Appl. Opt. 31, 6212-6219 (1992).

3. T. Mazurenko, "Time-domain Fourier-transform holography and possible applications in signal processing," Opt. Eng. 31, 739-749 (1992).

4. S. A. Akhmanov, A. S. Chirkin, K. N. Drabovich, A. I. Kovrigin, R. V. Khokhlov, and A. P. Sukhorukov, "Nonstationary nonlinear optical effects and ultrafast light pulse formation," IEEE J. Quantum Electron. QE-4, 598-605 (1968).

5. A. Papoulis, "Pulse compression, fiber communication, and diffraction: a unified approach," J. Opt. Soc. Am. A 11, 3-13 1994).

6. B. H. Kolner, "Space-time duality and the theory of temporal imaging," IEEE J. Quantum Electron. 30, 1951-1963 (1994).

7. B. H. Kolner and M. Nazarathy, "Temporal imaging with a time lens," Opt. Lett. 14, 630-632 (1989).

8. B. H. Kolner, "Active pulse compression using an integrated electro-optic phase modulator," Appl. Phys. Lett. 52, 11221124 (1988).

9. A. VanderLugt, Optical Signal Processing (Wiley, New York, 1992).

10. G. P. Agrawal, Nonlinear Fiber Optics (Academic, New York, 1989), Chap. 3.

11. H. G. Winful, "Pulse compression in optical fiber filters," Appl. Phys. Lett. 46, 527-529 (1985). 